\title{
Functionalized 2d Nano-Graphene Oxide Against Corona Virus Outbreak
}

\author{
Diogo José Horst* \\ Department of Mechanical Engineering and Sciences (pós-ECM), Federal University \\ of Santa Catarina (UFSC), Joinville, SC, Brazil \\ *Corresponding Author: Diogo José Horst, Department of Mechanical Engineering \\ and Sciences (pós-ECM), Federal University of Santa Catarina (UFSC), Joinville, SC, \\ Brazil.
}

The world is alarmed by the new viral epidemics such as the novel 2019-20 Wuhan coronavirus outbreak that can be lethal.

Coronaviruses are from the subfamily Orthocoronavirinae in the family Coronaviridae, in the order Nidovirales. Coronaviruses are enveloped viruses with a positive-sense single-stranded RNA genome and with a nucleocapsid of helical symmetry. The genomic size of coronaviruses ranges from approximately 26 to 32 kilobases, the largest for an RNA virus [1].

Coronaviruses primarily infect the upper respiratory and gastrointestinal tract of mammals and birds. They also cause a range of diseases in farm animals and domesticated pets, some of which can be serious and are a threat to the farming industry. In chickens, the infectious bronchitis virus (IBV), a coronavirus, targets not only the respiratory tract but also the urogenital tract. The virus can spread to different organs throughout the chicken [2].

Bioinspired synthesis offers potential green strategies to build highly complex nanomaterials by utilizing the unique nanostructures, functions, and properties of biomolecules, in which the biomolecular recognition and self-assembly processes play important roles in tailoring the structures and functions of bioinspired materials. Further understanding of biomolecular self-assembly for inspiring the formation and assembly of nanoparticles would promote the design and fabrication of functional nanomaterials for various applications [3].

Graphene oxide (GO) can efficiently capture viruses, destroy their surface proteins, and extract viral RNA in an aqueous environment by using the superficial bioreduction of GO. It follows from these phenomena that GO is an excellent nanomaterial for the high-throughput detection and disinfection of viruses, dem-
Received: February 14, 2020

Published: March 06, 2020

(C) All rights are reserved by Diogo José Horst. onstrating its great potential for the prevention of environmental infections [4].

Currently, abundant efforts have been devoted to research on graphene in interdisciplinary applications due to their unique physicochemical, electronic and biological properties. Accompanying the rapid developments of graphene, it is highly important to explore graphene based bio-functional nanostructures (G-BFNs) to bridge the gap between the fundamental advantages of graphene and the requirements in designing hybridized biomaterials [5].

As resistance to traditional drugs emerges for treatment of virus infections, the need for new methods for virus inhibition increases. Graphene derivatives with large surface areas have shown strong activity against different viruses. However, the inability of current synthetic protocols to accurately manipulate the structure of graphene sheets in order to control their antiviral activity remains a major challenge [6].

2D nanomaterials, particularly graphene, offer many fascinating physicochemical properties that have generated exciting visions of future biological applications [7].

2D nanomaterials could cause structural disruption and cytotoxic effects to cells, which greatly challenges their promising biomedical applications including biosensing, bioimaging, and drug delivery [8]. 2D materials can mechanically insert into cell membranes and extract lipids out, thus leading to the destruction of cell integrity. On the one hand, the cytotoxicity of 2D materials can be harnessed in surface engineering to resist biofouling, and on the other hand, it causes great concern with In vivo biomedical applications ranging from drug delivery to nanoimaging [9]. 
Graphene and its derivatives have recently attracted much attention for sensing and deactivating pathogens. However, the mechanism of multivalent interactions at the graphene-pathogen interface is not fully understood. Since different physicochemical parameters of graphene play a role at this interface, control over graphene's structure is necessary to study the mechanism of these interactions [10].

A summary of recent advances in the biocompatibility, toxicology and applications of graphene-based nanomaterials in biosystems is presented in figure 1 :

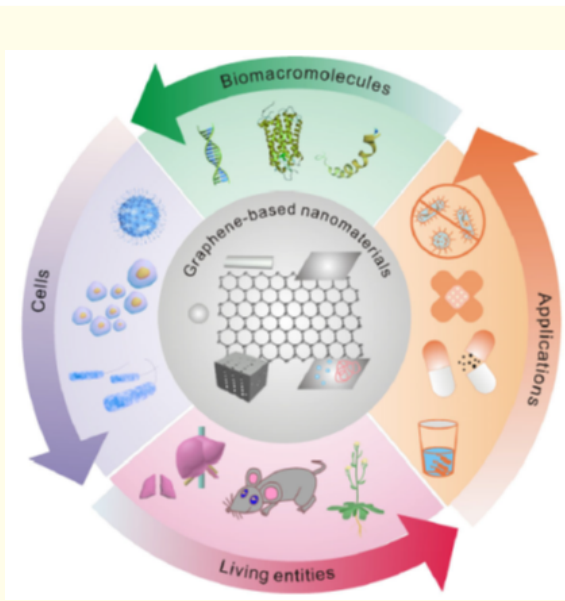

Figure 1: Overview of the biocompatibility, toxicology and applications of graphene-based nanomaterials in biosystems.

Source: [11].

Functional graphene nanomaterials (FGNs) are fast emerging materials with extremely unique physical and chemical properties and physiological ability to interfere and/or interact with bioorganisms; as a result, FGNs present manifold possibilities for diverse biological applications. Beyond their use in drug/gene delivery, phototherapy, and bioimaging, recent studies have revealed that FGNs can significantly promote interfacial biointeractions, in particular, with proteins, mammalian cells/stem cells, and microbials. FGNs can adsorb and concentrate nutrition factors including proteins from physiological media. This accelerates the formation of extracellular matrix, which eventually promotes cell colonization by providing a more beneficial microenvironment for cell adhesion and growth. Furthermore, FGNs can also interact with cocultured cells by physical or chemical stimulation, which significantly mediate their cellular signaling and biological performance [12].

In a recent study, Deokar., et al. [13] showed the design and synthesize sulfonated magnetic nanoparticles functionalized with reduced graphene oxide (SMRGO) to capture and photothermally destroy herpes simplex virus type 1 (HSV-1).

In another important contribution, remarkable observations on the adsorption and desorption mechanisms of single-stranded oligonucleotides and the hybridization of double-stranded DNA (ds-DNA) on a graphene oxide (GO) surface have been made using ensemble and single-molecule fluorescence methods. Probe and target DNAs labeled individually with fluorescence resonance energy transfer (FRET) pairs and having similar adsorption affinities toward the GO surface are used to provide detailed insights into the hybridization mechanism. Single-molecule FRET results reveal an "in situ" DNA hybridization mechanism, i.e., hybridization between the probe and target DNAs to form a ds-DNA, and simultaneous desorption from the GO surface thereafter. The results demonstrate that the electrostatic interaction between DNA and GO is of little importance to the overall theory of interaction and the largest effects are from solvation forces, specifically the hydrophobic effect. This investigation improves the fundamental understanding of the DNA hybridization dynamics on the GO surface, opening new windows in the field of biophysics as well as in sensing and therapeutic applications [14].

Polysulfated nanomaterials that mimic the extracellular cell matrix are of great interest for their potential to modulate cellular responses and to bind and neutralize pathogens. However, control over the density of active functional groups on such biomimetics is essential for efficient interactions, and this remains a challenge [15].

The combination of biomolecules with graphene-based materials offers a promising method to fabricate novel graphene-biomolecule hybrid nanomaterials with unique functions in biology, medicine, nanotechnology, and materials science [16].

Thus, the study and application of 2D functionalized nanomaterials especially using graphene oxide is of great interest to the scientific community in the fight towards the eradication of novel coronaviruses. 


\section{Bibliography}

1. De Groot RJ., et al. "Family Coronaviridae". In AMQ King, E Lefkowitz, MJ Adams, EB Carstens (eds.). Ninth Report of the International Committee on Taxonomy of Viruses (2011).

2. Bande F., et al. "Progress and challenges toward the development of vaccines against avian infectious bronchitis". Journal of Immunology Research (2015): 1-12.

3. Coucong Gong., et al. "Hierarchical nanomaterials via biomolecular self-assembly and bioinspiration for energy and environmental applications". Nanoscale (2019).

4. Song Z., et al. "Virus Capture and Destruction by Label-Free Graphene Oxide for Detection and Disinfection Applications". Small 11 (2015): 1171-1176.

5. Chuanxiong Nie., et al. "Recent progresses in graphene based bio-functional nanostructures for advanced biological and cellular interfaces". Nano Today (2019).

6. Ievgen S Donskyi., et al. "Mohsen Adeli and Rainer Haag, Functionalized nanographene sheets with high antiviral activity through synergistic electrostatic and hydrophobic interactions". Nanoscale (2019).

7. Zhaoxu Tu., et al. "Multivalent Interactions between 2D Nanomaterials and Biointerfaces". Advanced Materials 30.33 (2018).

8. Zhen Li., et al. "Modeling Interactions between Liposomes and Hydrophobic Nanosheets”. Small 15.6 (2018a).

9. Zhen Li., et al. "Temperature-Dependent Lipid Extraction from Membranes by Boron Nitride Nanosheets". ACS Nano (2018b).

10. Kok H Tan., et al. "Functionalized 2D nanomaterials with switchable binding to investigate graphene-bacteria interactions". Nanoscale (2018).

11. Na Lu., et al. "Graphene-based nanomaterials in biosystems". Nano Research (2018).

12. Chong Cheng., et al. "Functional Graphene Nanomaterials Based Architectures: Biointeractions, Fabrications, and Emerging Biological Applications". Chemical Reviews (2017).

13. Archana R. Deokar., et al. "Hot Plate" for the Capture and Destruction of the Herpes Simplex Virus Type 1". Bioconjugate Chemistry (2017).
14. Tapas Paul., et al. "Mishra, Single-Molecule FRET Studies of the Hybridization Mechanism during Noncovalent Adsorption and Desorption of DNA on Graphene Oxide". The Journal of Physical Chemistry $B$ (2016).

15. MF Gholami., et al. "Functionalized Graphene as Extracellular Matrix Mimics: Toward Well-Defined 2D Nanomaterials for Multivalent Virus Interactions". Advanced Functional Materials 27.15 (2017).

16. Dapeng Li., et al. "When biomolecules meet graphene: from molecular level interactions to material design and applications" Nanoscale (2016).

\section{Assets from publication with us}

- Prompt Acknowledgement after receiving the article

- Thorough Double blinded peer review

- Rapid Publication

- Issue of Publication Certificate

- High visibility of your Published work

Website: https://www.actascientific.com/

Submit Article: https://www.actascientific.com/submission.php Email us: editor@actascientific.com

Contact us: +919182824667 